\title{
Ultrasound-Mediated Gene and Drug Delivery Using a Microbubble-Liposome Particle System
}

\author{
Young Il Yoon ${ }^{1,2,3 *}$, Yong-Su Kwon ${ }^{*}$, Hee-Sang Cho ${ }^{4}$, Sun-Hee Heo ${ }^{5}$, Kyeong Soon Park ${ }^{5}$, Sang Gyu Park ${ }^{5}$, \\ Soo-Hong Lee ${ }^{5}$, Seung Il Hwang, 1,2, Young Il Kim¹, Hwan Jun Jae', Gook-Jun Ahn', Young-Seok Cho ${ }^{8}$, \\ Hakho Lee ${ }^{9 凶}$, Hak Jong Lee ${ }^{1,2,3,10 \bowtie}$, Tae-Jong Yoon ${ }^{\natural}$ \\ 1. Department of Radiology, Seoul National University College of Medicine, Seoul 110-799, South Korea; \\ 2. Department of Radiology, Seoul National University Bundang Hospital, Seungnam 463-707, South Korea; \\ 3. Program in Nano Science and Technology, Department of Transdisciplinary Studies, Seoul National University Graduate School of Convergence \\ Science and Technology, Suwon 443-270, South Korea; \\ 4. Department of Applied Bioscience, College of Life Science, CHA University, Pocheon 135-081, South Korea; \\ 5. Department of Biomedical Science, College of Life Science, CHA University, Pocheon 135-081, South Korea; \\ 6. Department of Radiology, Seoul National University Hospital, College of Medicine, Seoul 110-744, South Korea; \\ 7. Laboratory animal center, KBIO Osong Medical Innovation Foundation, Osong, Cheongwon, Chungbuk 363-951, South Korea; \\ 8. Department of Gastroenterology, Uijeongbu St. Mary's Hospital, College of Medicine, The Catholic University of Korea, Uijeongbu 480-717, \\ South Korea. \\ 9. Center for Systems Biology, Massachusetts General Hospital, 185 Cambridge St, CPZN 5206, Boston, MA 02114, USA. \\ 10. Nanoimaging and Therapy Research Center, Institute of Nanoconvergence, Advanced Institutes of Convergence Technology, Seoul National \\ University. \\ *These authors contributed equally to this work.
}

$\square$ Corresponding authors: Tae-Jong Yoon, PhD. Department of Applied Biosciences, College of Biomedical Sciences, CHA University, Pocheon 135-081, South Korea. +82-31-725-8389. tjyoon@cha.ac.kr Hakho Lee, PhD. Center for Systems Biology, Massachusetts General Hospital, 185 Cambridge St, CPZN 5206, Boston, MA 02114, USA. 1-617-643-3220, hlee@mgh.harvard.edu Hak Jong Lee, MD, PhD. Department of Radiology, Seoul National University, College of Medicine and Seoul National University Bundang Hospital, College of Medicine, Seongnam 463-707, South Korea. +82-31-787-7605. hakjlee@snu.ac.kr.

() Ivyspring International Publisher. This is an open-access article distributed under the terms of the Creative Commons License (http://creativecommons.org/ licenses/by-nc-nd/3.0/). Reproduction is permitted for personal, noncommercial use, provided that the article is in whole, unmodified, and properly cited.

Received: 2014.06.19; Accepted: 2014.08.08; Published: 2014.09.05

\begin{abstract}
Theranostic agents present a promising clinical approach for cancer detection and treatment. We herein introduce a microbubble and liposome complex (MB-Lipo) developed for ultrasound (US) imaging and activation. The MB-Lipo particles have a hybrid structure consisting of a MB complexed with multiple Lipos. The MB components are used to generate high echo signals in US imaging, while the Lipos serve as a versatile carrier of therapeutic materials. MB-Lipo allows high contrast US imaging of tumor sites. More importantly, the application of high acoustic pressure bursts MBs, which releases therapeutic Lipos and further enhances their intracellular delivery through sonoporation effect. Both imaging and drug release could thus be achieved by a single US modality, enabling in situ treatment guided by real-time imaging. The MB-Lipo system was applied to specifically deliver anti-cancer drug and genes to tumor cells, which showed enhanced therapeutic effect. We also demonstrate the clinical potential of MB-Lipo by imaging and treating tumor in vivo.
\end{abstract}

Key words: Microbubble; Liposome; Ultrasound imaging; Drug and gene delivery; Theranostics.

\section{Introduction}

Theranostic particles, which have a dual capacity for imaging and therapeutics, have emerged as a novel concept for cancer detection and treatment [1,2]. The approach could overcome intrinsic mismatch of key metrics (e.g., pharmacokinetics, selectivity, biodistribution) between imaging and therapeutic agents, thereby allowing for precise, imaging-guided drug delivery. The technology could also open up a new opportunity for rapid assessment and adjustment of treatment regime, based on real-time imaging feedback during therapy. Many different types of theranostic agents have been developed using mag- 
netic nanoparticles, quantum dots or novel metal nanoparticles as substrates [3, 4]. Existing detection modalities (e.g., magnetic resonance imaging, optical excitation) could be used to image these agents with high contrast, and often to actuate them for drug release. Most of the developed theranostic agents, however, are still difficult to be translated into clinical applications. A significant concern is the potential toxicity of constituent materials (e.g., metal or semiconductor nanoparticles) [5, 6]. Some agents also require specialized equipments, besides imaging instruments, to release therapeutic materials (e.g., magnetic hyperthermia, photodynamic therapy). Microbubble (micro-meter sized bubble, $\mathrm{MB}$ ) and liposome (Lipo) particles, conversely, are promising clinical application materials comprising non-toxic lipid chemicals. These newly developed materials can be used for the diagnosis and treatment of cancer and serve as alternatives to conventional theranostics that have known toxicological problems [7-11]. Synthesized with biocompatible polymers, these particles are shown to be non-toxic for in vivo uses. They can also be loaded with contrast agents for enhanced imaging (e.g., microscopy, MRI, ultrasound), and importantly, with therapeutic materials to treat tumor [12-17].

Kheirolomoon A. et. al. reported the first therapeutic use of microbubbles conjugated with liposomes encapsulating drugs in their hydrophilic core. They also used the ultrasound acoustic focusing to enhance the drug delivery to cells [18]. Albeit pioneering, the scope of the work for cancer therapy was limited: the therapeutic efficacy was low due to the non-specific delivery of the construct, and the application was only demonstrated in cell culture. The goal of this study is to further advance lipid-based particles for maximal treatment efficacy. In particular, we herein report a new particle construct 1) that can simultaneous deliver therapeutic drugs and genes into target cells with high specificity, and 2) that can be imaged and activated by US. We selected US both for imaging and drug/gene delivery mechanism, as it is fast, non-invasive and deep tissue penetrating. Furthermore, compared to other diagnostic imaging modalities, the US system is safe, cost-effective and routinely available in most clinics. In designing theranostic US particles, we adopted a hybrid approach: 1) MB, gas-filled lipid microsphere, was employed to enhance US imaging contrast; 2) Lipo was prepared, that encapsulated therapeutic drugs and genes; and 3) multiple Lipos were linked to a MB to form a MB-Lipo complex. The resulting particles generated high contrast under normal US illumination, as MBs oscillated and generated acoustic waves. For the delivery of therapeutic material, we burst MBs in situ by applying higher acoustical pressure; this unloaded Lipos from
MBs as well as temporarily permeabilized cellular membrane for efficient Lipo delivery [19-21]. The particle synthesis and loading of therapeutic agents were optimized. The prepared particles were imaged and activated using a conventional US scanner. We also confirmed that MB burst indeed improved the co-delivery of therapeutic agents. The clinical utility of MB-Lipo for US theranostics was evaluated in vivo by treating tumor in an animal model.

\section{Materials and Methods}

\section{Preparation of MBs}

All phospholipids compounds were purchased from Avanti Polar Lipids (Albaster, AL, USA) and used without any purification. 1,2-Dipalmitory-snglycero-3-phophatidylcholine (DPPC, $15.4 \mathrm{mg}$ ), dicetyl phosphate (DCP, $1.0 \mathrm{mg})$, 1,2-dipalmitorysn-glycero-3-phosphoethanolamine (DPPE, $1.2 \mathrm{mg}$ ), 1,2-distearoyl-sn-glycero-3-phosphoethanolamine-N-[ PDP(polyethylene glycol)-2000] (DSPE-PEG-PDP, 5.0 $\mathrm{mg})$, and cholesterol $(3.5 \mathrm{mg}$ ) were dissolved in $5 \mathrm{~mL}$ chloroform (99.9 \%, Sigma-Aldrich, St. Louis, MO, USA). To remove the solvent, the solution was evaporated for $5 \mathrm{~min}$ at $35^{\circ} \mathrm{C}$ and freeze-dried for $24 \mathrm{hr}$ at $-45^{\circ} \mathrm{C}$; this led to the formation of a phospholipid film. To create a lipid solution, a 2-mL mixture of glycerin, propylene, and $\mathrm{H}_{2} \mathrm{O}$ (1:2:7, volume ratios) was added to the film and transferred to a hermetic vial (Wheaton, NJ, USA). MBs were prepared via a mechanical mixing with a high-speed shaking-device (KIMS, South Korea) and $\mathrm{SF}_{6}$ gas filling. Fluorescein isothiocyanate (FITC, $1 \mathrm{mg}$, Sigma-Aldrich) was added to the lipid solution before the mixing for incorporation into the MB. Unloaded FITC was washed by centrifugation ( $5 \mathrm{~min}, 13,000 \mathrm{rpm}$ ). The MB particle concentration (C) was measured by AccuSizer (780/APS), and the weight $(W)$ of MB particles was measured from particle precipitates $(13,000 \mathrm{rpm}, 5$ $\mathrm{min})$. We then calculated a weight conversion factor ( $a$ $=W / C$ ). In our synthesis setup, $a=0.96 \times 10^{-11} \mathrm{mg} \cdot \mathrm{mL}$. For the subsequent assays, we measured MB concentrations and used the conversion factor to estimate the MB weight.

\section{Preparation of liposome particles}

The Lipo particles were produced in a similar manner. DPPC (15.4 mg), DCP (1.0 mg), DPPE (1.2 $\mathrm{mg}$ ) and cholesterol $(3.5 \mathrm{mg})$ were mixed in $5 \mathrm{~mL}$ chloroform. A lipid film was formed after evaporation $\left(5 \mathrm{~min}, 35^{\circ} \mathrm{C}\right)$ and lyophilization $\left(24 \mathrm{hr},-45^{\circ} \mathrm{C}\right)$. Two milliliters of $\mathrm{H}_{2} \mathrm{O}$ was added to the film, and the mixture was treated with sonication for $5 \mathrm{~min}$ at $60^{\circ} \mathrm{C}$. To make fluorescent Lipos, hydrophilic organic dye (Texas red, $1 \mathrm{mg}$, Sigma-Aldrich) was added to the 
film solution, and excess dye was removed by centrifugation ( $5 \mathrm{~min}, 13,000 \mathrm{rpm}$ ). The film solution was subjected to freezing and defrosting cycles (5 times) in liquid nitrogen and a water bath, respectively. The liposomal dispersion was extruded through a 200-nm filter using a mini-extruder at $60{ }^{\circ} \mathrm{C}$ (Avanti polar lipids, Albaster, AL). To add sulfhydryl functional group on the Lipo surface, amine-active Lipo, derived from DPPE, was treated with $5 \mathrm{mg}$ 2-iminothiolane $\cdot \mathrm{HCl}$ (Traut's reagent, Pierce) for $2 \mathrm{hr}$ at $25^{\circ} \mathrm{C}$ after adjusting the $\mathrm{pH}$ to 8 with $1 \mathrm{M} \mathrm{NaHCO}_{3}$.

\section{Preparation of MB-Lipo complex}

$0.67 \mathrm{~mL}$ Thiol-active MBs $\left(13 \mathrm{mg} / \mathrm{mL}, 1.2 \times 10^{11}\right.$ $\mathrm{MB} / \mathrm{mL}$ ), derived from DSPE-PEG-PDP, were mixed with $2 \mathrm{~mL}$ thiolated Lipo $(10 \mathrm{mg} / \mathrm{mL})$ by shaking for 2 $\mathrm{hr}$ at room temperature. The reaction was monitored to pyridine-2-thione releasing by UV-Vis spectra. The prepared Lipo still had an amine functional group on the surface, despite treatment with Traut's reagent, as shown by quantitative amine analysis. The $30 \mathrm{mg}$ MB-Lipo dispersion aqueous solution (2 $\mathrm{mL})$, containing an amine functional group, was reacted with sulfosuccinimidyl-4-(N-maleimidomethyl)cyclo hexane-1-carboxylate (sulfo-SMCC, $5 \mathrm{mg}$, SigmaAldrich) for $3 \mathrm{hr}$ at $25^{\circ} \mathrm{C}$ after adjusting the $\mathrm{pH} \sim 8.2$ with $1 \mathrm{M} \mathrm{NaHCO}_{3}$, to activate maleimide functional group. This particle solution was then linked to appropriate antibodies using a general conjugation procedure.

\section{Doxorubicin (Dox), plasmid DNA, and siRNA loading into MB-Lipo}

Doxorubicin (Sigma-Aldrich) was incorporated into Lipo particles by the thin-film hydration and remote-loading method with an ammonium sulfate gradient. Lipid film $(21.1 \mathrm{mg})$ was hydrated with 2 $\mathrm{mL}$ ammonium sulfate solution $(250 \mathrm{mM})$, and the liposomal suspension was sequentially extruded 5 times through polycarbonate filters with pore sizes of $200 \mathrm{~nm}$. The ammonium sulfate was removed by centrifugation (5 min, 13,000 rpm). At this point, ammonium sulfate ions were located only inside of Lipo particles. The liposomal dispersion and $440 \mu \mathrm{M}$ Dox $(1: 1, \mathrm{v} / \mathrm{v})$ were mixed and incubated for $2 \mathrm{hr}$ at 60 ${ }^{\circ} \mathrm{C}$. The mixture was washed to remove unloaded Dox, and the loading capacity of Dox in Lipo was determined by measuring the concentration of Dox in the supernatant by UV-Vis spectroscopy. Lipo Dox particles were then complexes with MBs as described above. To package plasmid GFP ( $p$ GFP) gene into Lipo, the Lipids film (21 mg) was lyophilized, and then homogenized with the complexes of $p$ GFP (10 $\mathrm{kbp}, 34 \mathrm{nM}$ ), and a 0.2-mL protamine (PA, $7.5 \mathrm{kD}, 18.8$ $\mu \mathrm{M})$ solution, at ratio that was optimized by electro- phoresis experiments was added to the Lipo powder (21 mg) and shaken for $5 \mathrm{~min}$ at $25^{\circ} \mathrm{C}$. The gene complex containing Lipo was washed 2 times through centrifugation (5 $\mathrm{min}, 13,000 \mathrm{rpm}$ ) to remove unloaded gene complex, and the loading capacity was measured by UV-Vis spectroscopy. Dox and therapeutic siRNA (siSTAT3, Thermo scientific) were incorporated into the Lipo particles simultaneously using the same procedures described above for Dox and $p$ GFP. All functional $20 \mathrm{mg}$ Lipo particles $(2 \mathrm{~mL}$ in PBS) were stored at $4{ }^{\circ} \mathrm{C}$ until further complexation with $0.67 \mathrm{~mL}$ of $\mathrm{MBs}\left(13 \mathrm{mg} / \mathrm{mL}, 1.2 \times 10^{11} \mathrm{MB} / \mathrm{mL}\right)$ and conjugation with antibodies. The anchored antibodies were quantitative analyzed by general protein assay methods [22].

\section{Phantom study for echogenicity of MB-Lipo particle system}

The echogenicity of MB-Lipo $(15 \mathrm{mg} / \mathrm{mL}, 1.6 \mathrm{x}$ $10^{11} \mathrm{MB} / \mathrm{mL}$ ) was evaluated using a phantom sample. The phantom was made using plastic connecting tube with inner diameter of $2 \mathrm{~mm}$, locating in the chamber filled with water. Ultrasound scanner (iU22, Philips, Bothell, WA, USA) equipped with 5- to $12-\mathrm{MHz}$ broadband linear probe was used for imaging.

\section{Transfection of $p$ GFP into cells using MB-Lipo}

The day before transfection, $3 \times 10^{4}$ cells were seeded in the wells of a 48-well plate (BD Falcon) or confocal 4-well plate (Lab-tex, NY, USA). HER2-MB-LipopgFP (DPPC $2.1 \mathrm{mM}, p$ GFP $0.68 \mathrm{nM}$ ) was added to cells in culture media containing $10 \%$ fetal bovine serum (FBS). Following $1 \mathrm{hr}$ incubation, MB-Lipo particles were washed away with PBS solution. For flash application, a clinical ultrasound scanner (iU22, Philips, Bothell, WA, USA) with a 5- to $12-\mathrm{MHz}$ broadband linear probe was used. The probe was placed in backside of the well plate, and flash pulses $(\mathrm{MI}=0.61)$ were applied for $1 \mathrm{~min}$. The cells were washed twice with culture media and cultured for 2 days.

\section{Cell cytotoxicity assay}

A 3-(4,5-dimethylthiazol-2-yl)-2,5-diphenyltetrazolium (MTT) assay kit (Invitrogen) was used to evaluate cell viability in the presence of MB-Lipo. SkBr3 cells (ATCC, VA, USA) were cultured in RPMI supplemented with FBS (10\%) and penicillin and streptomycin $(1 \%)$. Cells were maintained at $37^{\circ} \mathrm{C}$ in a humidified atmosphere containing $5 \% \mathrm{CO}_{2}$. At confluence, the cells were washed, trypsinized, and re-suspended in culture media. Cells were then seeded at a concentration of 5,000 cells/well in a 96-well tissue culture plate and allowed to grow overnight at $37{ }^{\circ} \mathrm{C}$ under $5 \% \mathrm{CO}_{2}$. Various MB-Lipo solutions were added at different concentrations into 
the culture media, and the cells were allowed to grow for another $24 \mathrm{hr}$. To test for cell viability, culture media were replaced with MTT solution. After $3 \mathrm{hr}$ of incubation at $37{ }^{\circ} \mathrm{C}$ under $5 \% \mathrm{CO}_{2}$, MTT solubilization solution was added to dissolve the resulting formazan crystals. Readings were measured spectrophotometrically at a wavelength of $570 \mathrm{~nm}$, with background absorbance at $690 \mathrm{~nm}$, to determine cell viability.

\section{Tumor rabbit model}

This study was approved by the Animal Care Committee of Seoul National University Hospital (IACUC No. 12-0315) and was performed in accordance with local ethical guidelines. A total of 9 adult New Zealand white rabbits (Biogenomics, South Korea), weighing $2.5-3.0 \mathrm{~kg}$ each, were used in this experiment. Rabbits were anesthetized with an intramuscular injection of ketamine hydrochloride (25 $\mathrm{mg} / \mathrm{kg}$; Ketalar, Yuhan Yanghang, South Korea) and $2 \%$ xylazine hydrochloride (10 mg/kg; Rompun, Bayer Korea). The abdomen of the rabbit was shaved and prepared with povidone iodine. Laparotomy was performed with a 2-cm left paramedian incision to expose the left kidney. An 18-gauge needle consisting of a cannula and a core was used for tumor implantation, and one VX2 tumor fragment was placed into the lumen of the cannula. The puncture needle was directly inserted into the sub-capsular parenchyma of the left kidney, and the tumor fragment in the lumen of the needle was pushed out the core [23]. After light pressure on the implantation point to prevent bleeding and leakage of tumor tissues, the left kidney was returned to the abdominal cavity, and the abdomen wall was closed in double layers. All the procedures were performed aseptically, and tumors were allowed to grow for 2-3 weeks to reach a size of $15-30 \mathrm{~mm}$ in diameter.

\section{Treatment with therapeutic MB-Lipo particle to the in vivo rabbit model}

Trans-catheter intra-arterial administration of 1 $\mathrm{mL}$ of HER2-MB-Lipo Dox+sistAT3 $\left(15 \mathrm{mg} / \mathrm{mL}, 1.6 \times 10^{11}\right.$ $\mathrm{MB} / \mathrm{mL}$ ) was performed under fluoroscopic guidance by a interventional radiologist who had $>10$ years of experience in the procedure. The same anesthesia protocol as with VX2 tumor implantation was used. An 18-gauge catheter (BD Angiocath Plus with intravenous catheter, Becton Dickinson South Korea) was inserted into the right central auricular artery for arterial access, and a 2.0-Fr micro-catheter (Progreat, Terumo, Japan) was advanced along the 18-gauge catheter to the descending aorta by way of the external carotid artery. The central auricular artery is the major artery supplying blood to the rabbit ear. It lies in the central region of each ear and runs along the rostral edge of the ear [24]. As the central auricular branch lies beneath a thin skin sheet, it can be used to detect the left renal artery. Thus, angiography was performed to identify the renal arterial anatomy and the branching arteries feeding the tumor. When the catheter was advanced to an adequate position in the left renal artery, the prepared $\mathrm{MB}$ solution was infused through the catheter at rate of $6.6 \mathrm{~mL} / \mathrm{min}(1.5$ min) using an infusion pump (Genie Plus, Kent Scientific Corporation, Torrington, CT, USA) to avoid retrograde reflux of the injected materials out of the renal artery. After completing the intra-arterial injection, the microcatheter was removed from the auricular artery, and the puncture site was compressed manually for $90 \mathrm{~s}$.

\section{Ultrasound imaging and applying of flash in vivo}

Before and during delivery of HER2-MB-Lipo through catheter, a genitourinary radiologist with > 20 years of experience in renal ultrasound imaging performed the ultrasound imaging. All ultrasound imaging was performed with an iU22 Ultrasound scanner (Philips Healthcare, Bothell, WA, USA) equipped with a 5- to $12-\mathrm{MHz}$ broadband linear transducer. One minute after starting of MB-Lipo injection through an infusion pump, flashes with mechanical index of 0.61 were performed during 5 minutes with pulse width of 3 seconds.

\section{Magnetic resonance imaging (MRI) of tumors}

The pre-procedural MR images were acquired to evaluate presence of tumor and its size. All images were acquired on a 3.0-T clinical MR scanner (MagnetomVerio; Siemens Healthcare, Erlangen, Germany) using an 8-channel knee coil (In vivo, Gainesville, FL, USA). T2-weighted coronal TrueFISP images were obtained covering the both kidneys with following parameters: repetition time (TR), $5.52 \mathrm{~ms}$; echo time (TE), 2.76 ms; matrix, 256×256; flip angle, 66 degrees; slice thickness, $2 \mathrm{~mm}$ ( 25 slices); number of averages, 2; bandwidth of $501 \mathrm{~Hz} /$ pixel; and the field of view (FOV), $150 \times 150 \mathrm{~mm}$. A T2-weighted axial turbo spin echo (TSE) images were also obtained by using following parameters: TR, $5645 \mathrm{~ms}$; TE, $87 \mathrm{~ms}$; matrix, 512×358; flip angle, 144 degrees; slice thickness, $3 \mathrm{~mm}$ (35 slices); echo train length (ETL), 14; number of averages, 2; bandwidth of $250 \mathrm{~Hz} /$ pixel; and the FOV, $130 \times 130 \mathrm{~mm}$. T1-weighted axial turbo spin echo (TSE) images were also obtained with TR, $628 \mathrm{~ms}$; TE, 9.1 ms; matrix, 512×369; flip angle, 140 degrees; slice thickness, $3 \mathrm{~mm}$ (35 slices); ETL, 4; number of averages, 2; bandwidth of $376 \mathrm{~Hz} /$ pixel; and the FOV, $130 \times 130 \mathrm{~mm}$. Additional axial and coronal 
T1-weighted images were obtained by using 3D Volumetric Interpolated Breath Hold Examination (VIBE) sequence with TR, $5.12 \mathrm{~ms}$; TE, $1.95 \mathrm{~ms}$; matrix, 320×320; flip angle, 15 degrees; slice thickness, $3 \mathrm{~mm}$ (axial) or $2.5 \mathrm{~mm}$ (coronal); number of averages, 2; bandwidth of $401 \mathrm{~Hz} /$ pixel; and the FOV, $150 \times 150 \mathrm{~mm}$. For imaging follow-up, all subjects underwent the same MR image sets by using the same imaging parameters.

\section{Results and Discussion}

\section{Preparation of functional MB-Lipo particles}

Microbubble (MB) and liposome (Lipo) particles were prepared in separate processes. We synthesized MB particles from phospholipids and cholesterol (Fig. 1A). A phospholipid film was produced by lyophilizing a mixture of phospholipids and cholesterol. The film was redissolved in aqueous buffer under mechanical vibration and $\mathrm{SF}_{6}$ gas bubbling, which resulted in the formation of MBs filled with hydrophobic $\mathrm{SF}_{6}$ gas. During this process, we also added a relative hydrophobic dye (fluorescein isothiocyanate/FITC) that was incorporated into the MB shells. The prepared green-fluorescent $\mathrm{MB}$ particles had a
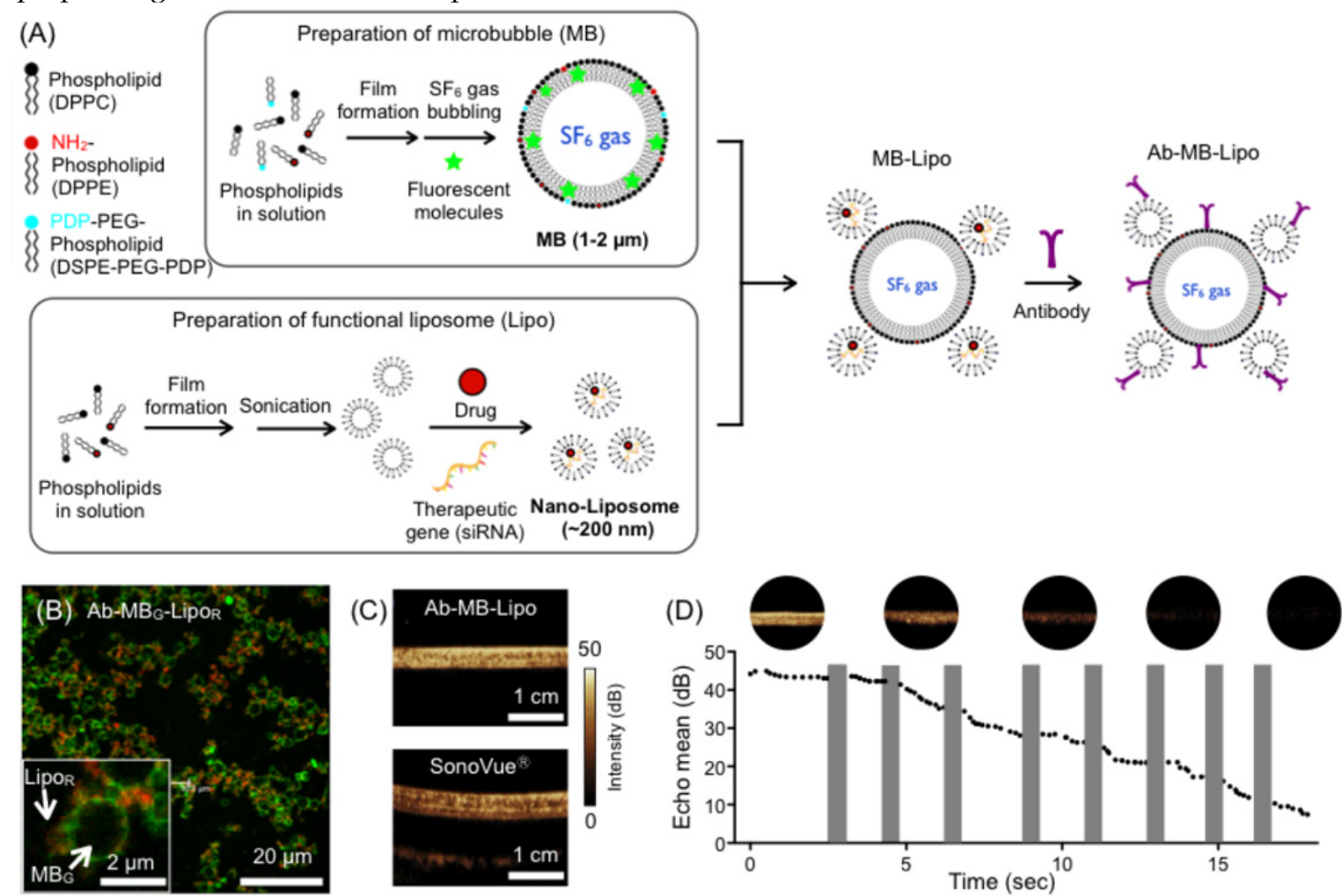

Figure I. Synthesis and characterization of microbubble (MB) and liposome (Lipo) complexes. (A) MB particles (average size $\sim 1.5 \mu \mathrm{m})$ were prepared from phospholipid compounds through mixing, film formation, and bubbling with $\mathrm{SF}_{6}$. Hydrophobic organic dyes could be incorporated into the MB membrane. Lipo particles (200 nm in diameter) were produced from phospholipid compounds through film formation and sonication. Hydrophilic dyes, pDNA, drugs, or therapeutic genes could be incorporated into the Lipo core. The prepared MB and Lipo particles were then assembled, and antibodies were conjugated. (B) Fluorescent MB-Lipo complexes were imaged. MBs were infused with hydrophobic dye (fluorescein isothiocyanate/FITC), whereas Lipos contained a hydrophilic dye (Texas Red). (C) Owing to SF 6 gas inside the MBs, Ab-MB-Lipo complexes generated high echogenicity under ultrasound (US) imaging ( $\mathrm{MI}=0.08$, imaging mode). The echogenicity signal intensity was similar to that with a commercial contrast agent (SonoVue $\left.{ }^{8}\right)$. (D) Higher acoustic pressure ( $\mathrm{Ml}=0.6 \mathrm{I}$; flash mode) burst MBs, which decreased the echo signal intensity. The signal from MB complex becomes negligible after 8 pulses application. The shaded bars indicate the duration when the flash pulse was applied. 
We next evaluated the MB-Lipo's echogenicity, i.e., the capability of the particles to return US signals. Phantom samples were prepared by filling plastic tubes with MB-Lipo or commercial agents (SonoVue ${ }^{\circledR}$, Bracco, Milan, Italy), and placed in a water bath. Samples were then imaged with a clinical US scanner (iU22, Philips, Bothell, WA, USA). The acoustic pressure, measured by the mechanical index (MI), was MI $=0.08$. MB-Lipo generated a high contrast against the background (Fig. 1C); its performance was comparable to that of SonoVue ${ }^{\circledR}$, which confirmed the feasibility of using MB-Lipo as US contrast agents. Furthermore, the particles maintained echogenicity for $>2$ weeks when stored in a gas-filled container (Supplementary Material: Fig. S3). At elevated acoustical pressure $(\mathrm{MI}=0.61$, flash mode), $\mathrm{MBs}$ underwent bursting disintegration, which could be observed by a decrease of their signal intensity. For example, the signal from MB-Lipo particles gradually reduced with consecutive application of flash pulses (Fig. 1D); signal disappeared after 8 pulses.

\section{Cancer cell targeting and enhanced intracellular delivery of Lipo particles}

MB-Lipo particles could be exploited to significantly improve the delivery of Lipo particles into cells; burst of MBs at the flash mode not only released the attached Lipo particles, but also enhanced the permeability of the cell membrane (sonoporation) for more efficient particle uptake (Fig. 2A) [25-27]. We first investigated such effects in vitro. Dual fluorescent MB-Lipo particles $\left(\mathrm{MB}_{\mathrm{G}}\right.$, green; $\mathrm{Lipo}_{\mathrm{R}}$, red) were prepared, and further conjugated with HER2 antibody $\left(\right.$ HER2-MB $\left.{ }_{\mathrm{G}}-\mathrm{Lipo}_{\mathrm{R}}\right)$. The particles were incubated with breast cancer cells $(\mathrm{SkBr} 3)$ that over-express HER2. Confocal microscopy showed a highly selective cell targeting (Fig. 2B). Before the application of US flash ( $t$ $=0)$, both green $(\mathrm{MB})$ and red (Lipo) fluorescence signals were observed on the outer cell membrane, as MB-Lipo particles were not taken up by the cells presumably due to the large size of MBs. The targeted cells were then exposed to US activation. We placed a 5- to $12-\mathrm{MHz}$ linear probe into the culture dish, and applied US pulses for $1 \mathrm{~min}(\mathrm{MI}=0.61$, flash mode). After the flash, we observed both green and red fluorescence inside the cells (Fig. 2C). With burst of MBs, both fluorescent dyes were released and subsequently transported into the cytoplasm. Note that MB-Lipos remained on the cellular membrane in the absence of US flash.

\section{Application of MB-Lipo as gene transfection agents}

We next utilized MB-Lipo particles as a gene transfection vehicle. We reasoned that the synergistic effect of MB bursting (i.e., Lipo release and sonoporation) would significantly enhance the transport of Lipos that carry target genes. To test this hypothesis, we transfected cancer cells with plasmid green fluorescent protein $(p \mathrm{GFP}, 10 \mathrm{kbp})$ genes. To increase $p$ GFP loading efficiency into the Lipos, we complexed the plasmid with protamine (PA, $7.5 \mathrm{kD})$ [28-30]. The optimal PA- $p$ GFP complex ratio was $20 \mu \mathrm{M}$ PA and $34 \mathrm{nM} p \mathrm{GFP}$ (600 PA molecules per $p \mathrm{GFP}$ ). The complex was efficiently loaded into the Lipo with $95 \%$ loading capacity which was prepared from this lipid film, and attached to MBs (Supplementary Material: Fig. S4). The MB-Lipo complex was further derivatized with antibodies (HER2-MB-Lipo $G F P$ ) for cell targeting. Figure 3 summarizes GFP gene transfection using the MB-Lipo particles. Cancer cells $(\mathrm{SkBr} 3)$ were treated with HER2-MB-Lipo ${ }_{\text {GFP }}(1 \mathrm{hr})$, followed by the application of US flash (MI $=0.61)$ for $1 \mathrm{~min}$ and culture. Control samples were prepared in a similar manner, but using particles without antibody (MB-LipogfP). Microscopy analyses (48 hrs post flash) confirmed target-specific gene transfection; we observed bright green fluorescence in cells targeted with HER2-MB-Lipo ${ }_{G F P}$, while the control had negligible signal. Furthermore, MB-Lipo particles displayed a higher transfection efficiency $(\sim 60 \%)$ than a commercial agent (Lipofectamine, Invitrogen; Supplementary Material: Fig. S5A and B). By changing the antibody, we could also transfect different cell types with high yields. For example, cardiac fibroblasts (CFts), which are known to be difficult to transfect as a primary cell line, expressed high levels of green fluorescence when targeted with CD29-specific MB-LipoGFP and stimulated with US flash (Fig. S5C).

\section{Therapeutic application of MB-Lipo as a delivery system for anticancer drugs and SiRNA}

The MB-Lipo system could serve as a versatile platform for disease treatment, as different therapeutic agents could be incorporated into liposomes. As a proof-of-concept, we prepared particles loaded with doxorubicin (Dox), an anticancer drug. Dox could be easily incorporated into the hydrophilic core of the Lipo particles using an ammonium sulfate gradient (see Methods) [31]. The Dox loading capacity was $88.7 \%$, as determined by UV-Vis spectroscopy. Following the 1-hour incubation of cancer cells (SkBr3) with HER2-MB-Lipo Dox (total Dox dose, $0.19 \mu \mathrm{M}$ ), we treated the cells with US flash $(\mathrm{MI}=0.61)$. The drug efficiently penetrated into the cell nucleus (Fig. 4A), leading to significant cell death (35\% viability, $72 \mathrm{hrs}$ post flash). In contrast, cells incubated with free Dox $(0.19 \mu \mathrm{M}, 1 \mathrm{hr})$ showed $90 \%$ viability when monitored 72 hours after the treatment. 
The MB-Lipo could also be used to deliver siRNA for therapeutic applications. As an example, we loaded Lipo with a tumor suppressor siRNA for STAT3 (signal transducer and activator of transcription 3), a transcription factor involved in cell proliferation $[32,33]$. As in the case with plasmid DNA, the siRNA for STAT3 (siSTAT3) was inserted into the Lipo particles after PA complexation (loading capacity, $95 \%)$. Target cells (SkBr3) were incubated with HER2-MB-Lipostat3, followed by US flashing. With the delivery of siSTAT3 to SkBr3 cells, the STAT3 gene was indeed down-regulated. We also observed the decrease of cyclin D1 and c-Myc gene expressions, which are involved in cell proliferation (Fig. 4B) [34, 35]. Such altered gene expression changed the cellular protein expression as well (Supplementary Material: Fig. S6). The levels of STAT-3 and cyclin D2 both decreased, whereas caspase3, an indicator of apoptosis, increased $[36,37]$. The overall cell viability after the siRNA delivery was $\sim 50 \%$ (72 hr post flash).

(A)

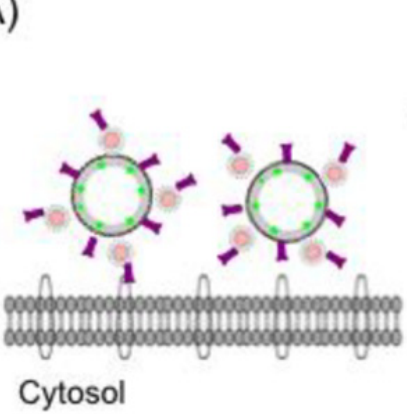

US flas

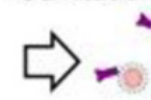

POP
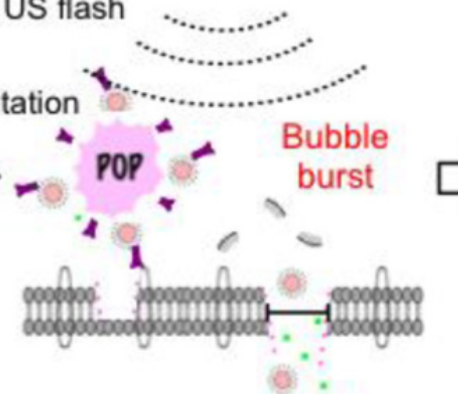

Bubble burst

(B)
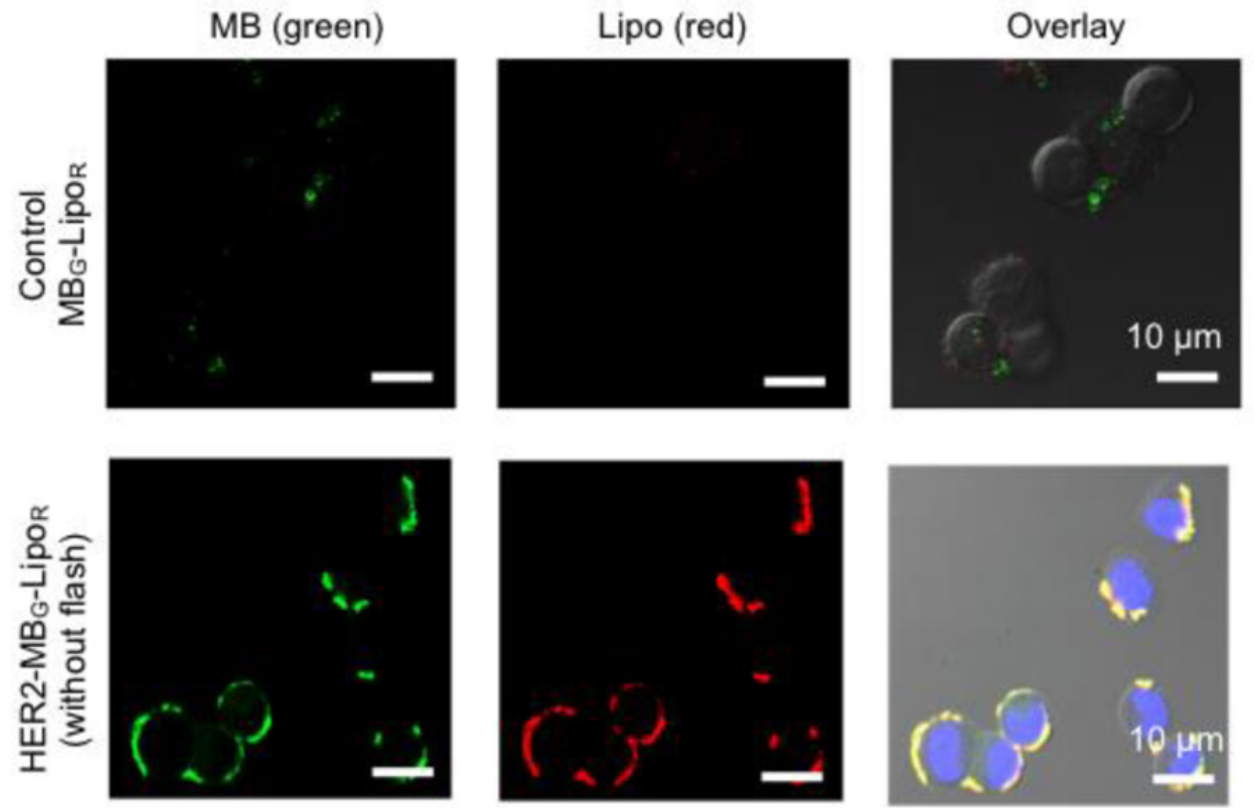

(C)
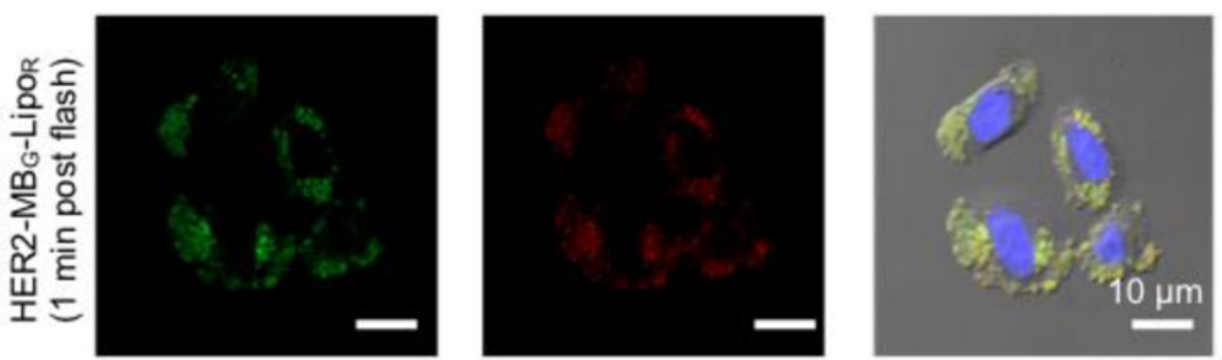

Figure 2. Specific and enhanced delivery of therapeutic agents with MB-Lipo particles. (A) Delivery scheme. Following specific targeting of MB-Lipo complexes (left), US pulses (flash mode) are applied to burst MBs and thereby release Lipos. The microjets and shockwaves from the MB burst also induce the formation of transient pores ( $220 \mathrm{~nm}$ diameter) on cell membrane, which facilitates the intracellular delivery of cargo material (middle). These temporary pores close in < I min (right). (B) Fluorescent

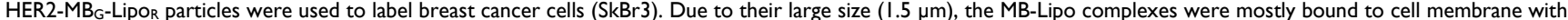
minimal intracellular uptake. (C) Application of US flash $(\mathrm{MI}=0.6 \mathrm{I})$ burst $\mathrm{MBs}$ and released Lipo particles, as confirmed by green and red fluorescent signal inside the cells. The uptake was fast with most of the organic dyes being taken up within I min of incubation. 

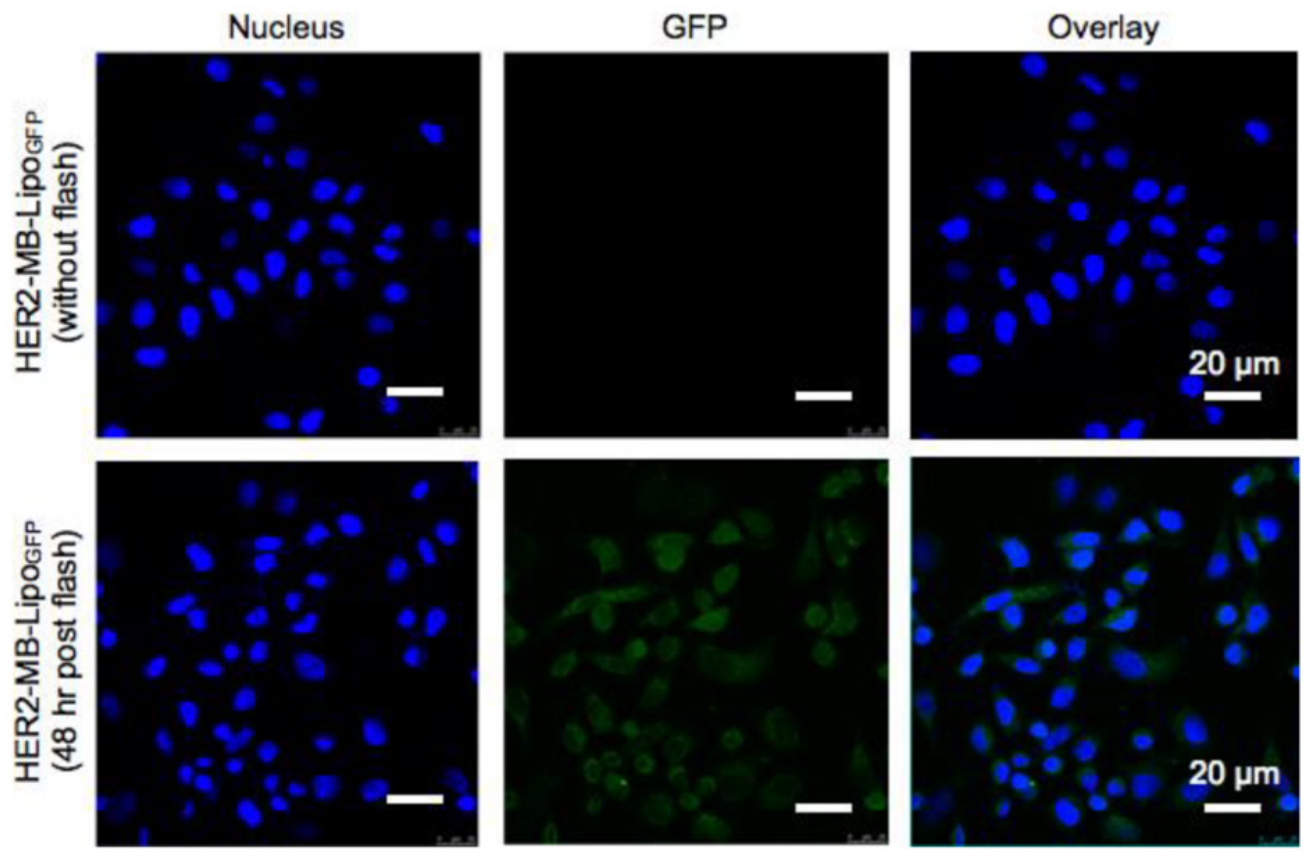

Figure 3. Plasmid gene delivery with MB-Lipo particles. MB-Lipo complexes containing fluorescent plasmid gene ( $p$ GFP) were prepared (HER2-MB-LipoGFP). Following target-specific labeling of cells ( $\mathrm{SkBr} 3)$, US flash was applied for I min to deliver Lipogfp into the cells ( $\mathrm{SkBr} 3)$. After $48 \mathrm{hr}$ culture, targeted cells showed green fluorescence (bottom row); the fluorescence signal was expressed throughout the whole cytoplasm, as is typical for GFP expression by $p$ GFP delivery. Non-flash treating cells as a control (top row) showed no GFP signal.
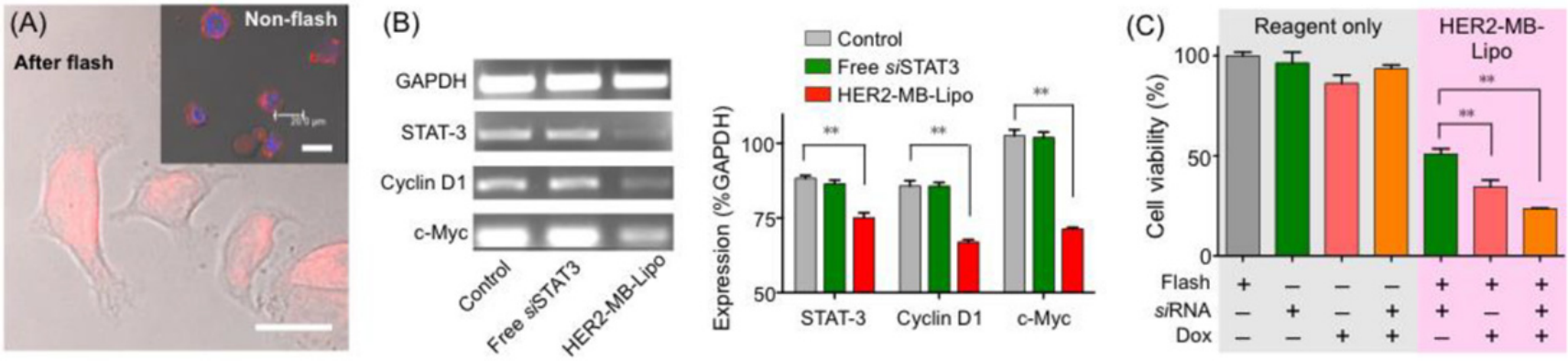

Figure 4. Delivery of therapeutic drug and siRNA. (A) Cancer cells ( $\mathrm{SkBr3}$ ) were targeted with HER2-MB-LipoDox particles that had Lipos loaded with doxorubicin (Dox). Following the flash treatment ( $\mathrm{MI}=0.6 \mathrm{I}, \mathrm{I} \mathrm{min}$ ) and $24 \mathrm{hr}$ culture, Dox (red) was observed to translocate into the nuclei. In control samples (inset) where cells were incubated with HER2-MB-LipoDox but did not receive flash pulses, most MB-Lipo particles remained on cell membrane even after 24 hr culture. Scale bars, $20 \mu$ m. (B) MB-Lipo particles were used to deliver a tumor suppressor siRNA for STAT3. In cancer cells (SkBr3) treated with HER2-MB-Lipo siSTAT3 and flash pulses, the level of STAT3 gene was lowered, as determined by polymerase chain reaction (PCR; left). Other cell proliferation gene markers (cyclin DI and c-Myc) were also found to be down-regulated. Control cells with only flash or siSTAT3 did not show altered STAT3 expression. All changes could be quantified and are statistically significant (right). (C) Cell viability was measured post I20 hr treatment with different therapeutic agents. Using the MB-Lipo as a delivery vehicle consistently led to a higher therapeutic efficiency, and MB-Lipos loaded with Dox and siSTAT3 demonstrated the highest potency in inducing cell death. Data are presented as mean \pm SE from triplicate measurements. $* * p<0.0 \mathrm{I}$.

Figure $4 \mathrm{C}$ summarizes the overall therapeutic efficacy of the MB-Lipo systems. Compared to free reagents (Dox, siSTAT3 or both), MB-Lipo agents combined with flash treatment displayed higher potency, which could be attributed to more efficient intracellular delivery of therapeutic agents. MB-Lipo particles co-delivering both Dox and siSTAT3 (see Methods) led to the lowest cell viability (25\%) from synergistic effect (Supplementary Material: Fig. S7).

\section{Theranostic application of MB-Lipo in vivo}

We finally applied MB-Lipo particles as theranostic agents in vivo. Namely, the particles were used to enhance US imaging contrast, as well as to enable US-mediated delivery of targeted gene and drug. For this demonstration, we established a rabbit renal tumor model by implanting VX2 tumor cells on the rabbit renal parenchyma (see Methods) [38]. The implanted tumor expressed HER2 as measured by immunohistochemistry and Western blotting (Supplementary Material: Fig. S8). We therefore used HER2-specific MB-Lipo for cell targeting, and loaded the particles with Dox and siSTAT3 for treatment. Prepared particles (HER2-MB-Lipo Dox+siSTAT3) were injected into the tumor sites through a catheter located in the left renal artery (see Methods). Following the intra-arterial (IA) particle injection, US imaging revealed a tumor region with high contrast proffered by 
MBs (Fig. 5A). Subsequently, we applied localized US pulses (flash, $\mathrm{MI}=0.61,10$ times) to burst MBs and deliver therapeutic-Lipos. The destruction of MBs could be monitored in situ by observing the loss of echogenicity in the targeted region (Fig. 5A, right).

The accumulation of HER2-MB-Lipo into tumor sites could be attributed to the combined effect of high permeability of tumor vasculature and active targeting. In tumor microenvironment, blood vessels are shown to be defective with large gaps between endothelial cells [39, 40]. MB-Lipo particles thus can leak from the vessels and enter into tumor interstitium. Coating MB-Lipo with antibodies further improves the retention of MB-Lipo in tumor via receptor-mediated active targeting [41].

Local co-delivery of therapeutic agents was validated by performing microscopy and polymerase chain reaction (PCR) analyses on the tissues extracted 4 days after US flash. The tumor tissue showed in- trinsic red fluorescence of Dox (Fig. 5B), and the expression of the target STAT3 gene was efficiently blocked by siSTAT3 (Fig. 5C). Therapeutic effect was negligible in control cohorts, which was either non-targeted with particles (only flash treatment) or targeted but with no flash applied.

We next sought to verify the therapeutic effect of MB-Lipo particles on tumor growth (see Methods). HER2-MB-Lipo Dox+sisTAT3 particles were administered into tumor sites on two separate days (day 1 and 5), and exposed to US-flash. MRI imaging was performed on days 0,4 and 10 to analyze the tumor size and growth rate (Fig. 6A). Indeed, tumors that were treated with MB-Lipo and US flash exhibited significantly decreased growth as compared to untreated tumors and tumors not exposed to flash (Fig. 6B). Histology data on excised tumor further confirmed that the cell density of the treated tumors was lower than those of controls (Fig. 6C).
(A)

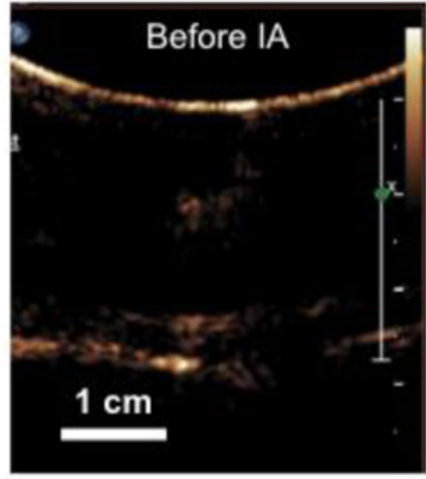

(B)
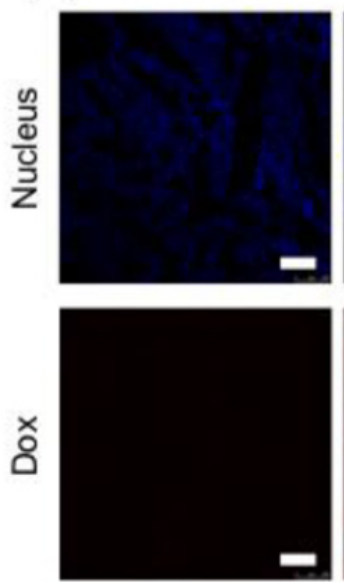

Non-treated (flash only)
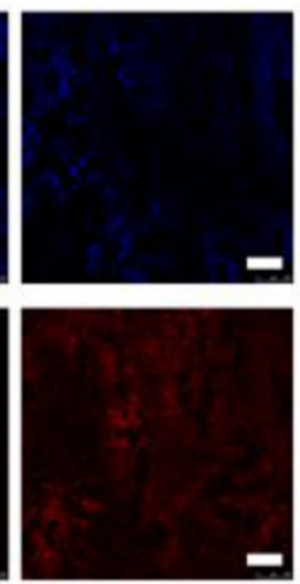

HER2-

MB-LipODox+sisTAT3 w/ flash
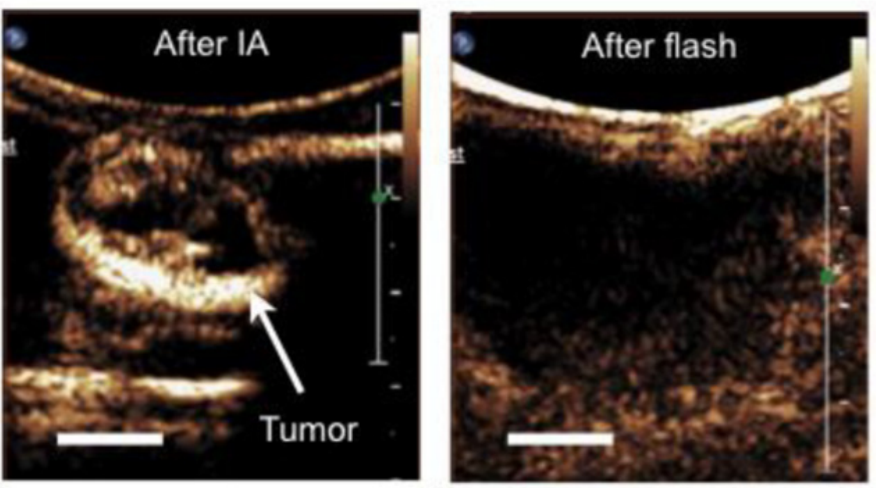

(C)
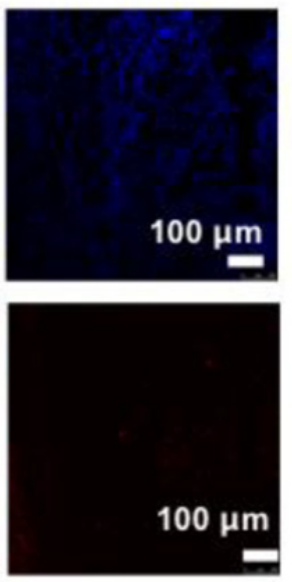

HER2-

MB-LipODox+siSTAT3 w/o flash
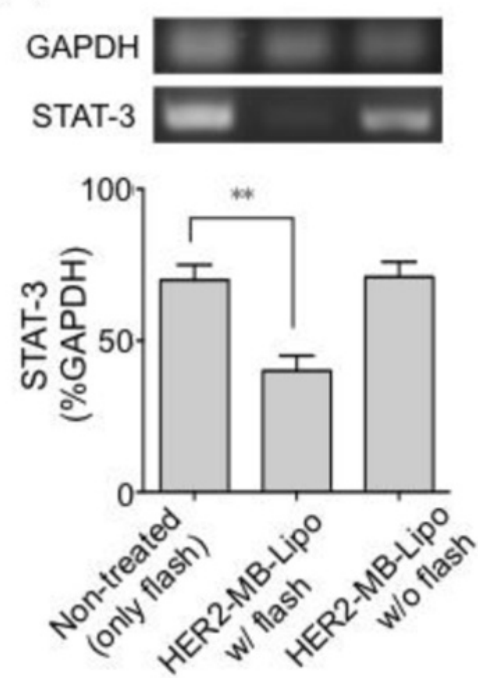

Figure 5. Target specific delivery of therapeutic drug and gene in vivo. (A) Therapeutic MB-Lipo particles containing both doxorubicin and siRNA (siSTAT3) were administered into the implanted tumor (VX2) region in a rabbit kidney via intra-articular (IA) injection. US imaging $(\mathrm{MI}=0.08)$ confirmed target-specific labeling of tumor with HER2-MB-LipoDoX+sistAT3 particles (middle). Concurrent with US tumor imaging, flash pulses were applied (MI $=0.6 \mathrm{I})$ to release therapeutic Lipos. MB burst could be confirmed by the disappearance of high echo signal (right). (B) Extracted tumor tissue was analyzed for drug delivery. The tumor treated with a combination of HER2-MB-LipoDox+sistAT3 and US flash (middle) showed high concentrations of Dox (red fluorescence). The signal was negligible in control samples (i.e., flash-only, MB-Lipo-targeted but without flash). Scale bar, $100 \mu \mathrm{m}$. (C) The delivery of siSTAT3 was confirmed by performing PCR on tissue extract. The treated tumor (HER2-MB-Lipodox+sisTAT3 and US flash) displayed a significant decrease $(* * p<0.01)$ in STAT3 expression as compared to controls. Each bar represents the mean \pm SE from triplicate measurements. 

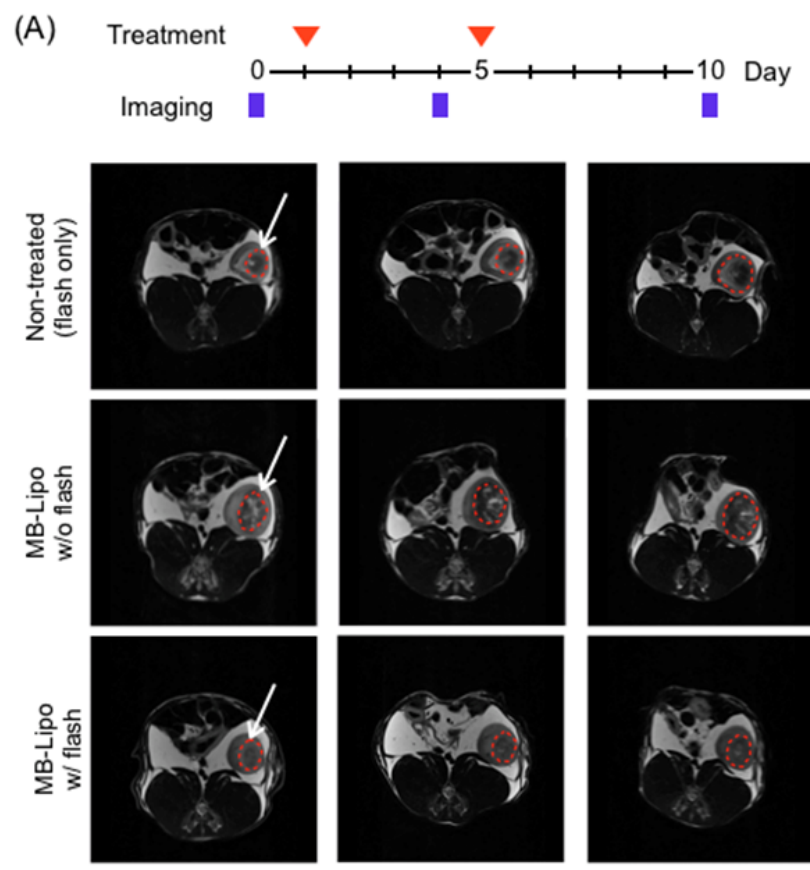

Day 0
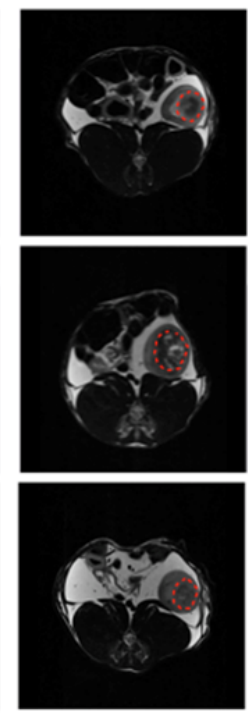

Day 4
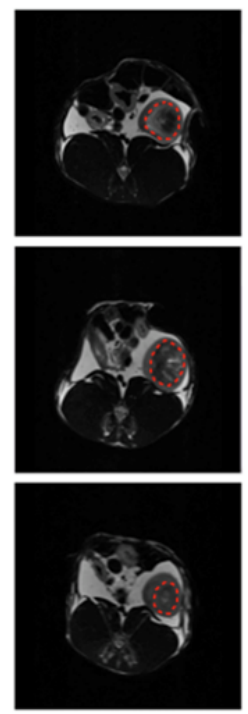

Day 10
(B)

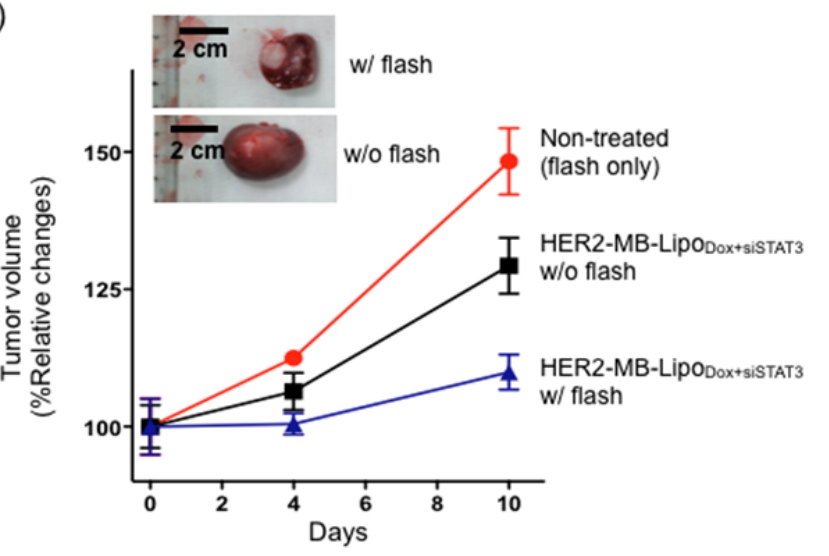

(c)

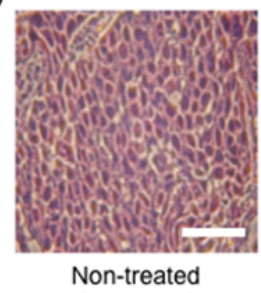

(flash only)

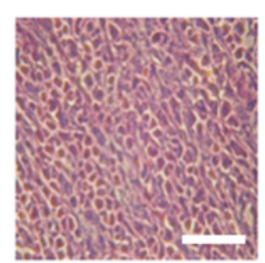

HER2-MB-LipoDox+siSTAT3 w/o flash

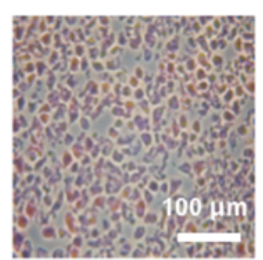

HER2-MB-LipoDox+siSTAT3 w/ flash

Figure 6. In vivo therapeutic application of MB-Lipo particles. (A) The efficacy of HER2-MB-LipoDox+sisTAT3 on tumor treatment was evaluated in vivo using a rabbit renal tumor model. For the treated group, MB-Lipo complexes were introduced on days I and 5 (red arrows) via intra-arterial injections. Half of the treated animal also received US flash for drug release. Tumor size was monitored by MRI imaging on days 0,4 , and 10. The dotted circles (red) indicate the VX2 tumor region. (B) Tumors treated with particles and flash showed markedly decreased growth rate. (Inset) Images of extracted tumors, demonstrating the therapeutic effects. (C) After treatment, histological changes in the implanted tumor tissues were evaluated by hematoxylin and eosin staining.

The bio-distribution of MB-Lipo after targeting and US flash was determined by analyzing the presence of fluorescent dyes in the liver, lungs, kidneys, and tumor (Supplementary Material: Fig. S9). For this purpose, we used dual fluorescent HER2-MB $\mathrm{G}_{\mathrm{G}}-\mathrm{Lipo}_{\mathrm{R}}$ particles, which were intravenous injected. Majority of the particles were found in the liver and lungs, independent of exposure to flash. However, following the application of flash at the tumor site, relative high-intensity fluorescence was observed within the tumor, which showed a 3-fold increase as compared to the non-flash control.

\section{Conclusions}

Theranostic agents are a promising clinical application strategy for accurate, facile disease detection and treatment method. We herein reported a hybrid theranostic agent for ultrasound (US), namely a microbubble and liposome complex (MB-Lipo) particle. Applying US for its detection as well as activation, we showed that the MB-Lipo platform has unique advantages over other theranostic modalities (e.g., photodyamic theraphy, magnetic hyperthermia). First, the detection modality enables real-time, deep tissue imaging, and the use of MBs significantly improves the signal contrasts. Second, the MB-Lipo particles can be remotely activated by the same US scanner (flash mode) during imaging; this capacity not only simpli- fies the required instrumentation, but also offers high spatiotemporal precision in treatment. Third, MB bursting with US pulses could amplify the intracellular delivery of therapeutic liposomes by temporarily increasing local cell membrane permeability (sonoporation). As a proof-of-concept, we have synthesized and systematically characterized the MB-Lipo particles. Both MBs and Lipos were produced with FDA-approved phospholipids, to maximize the product's biocompatibility for in vivo applications. As a contrast agent, the MB-Lipo particles yielded high US signal, similar to that of a commercial agent $\left(\right.$ SonoVue ${ }^{\circledR}$. Enhanced drug delivery through US activation (flash pulses) was also validated. Importantly, the MB-Lipo complex was proven to be a versatile platform for multi-functional operation. It can be rendered highly target-specific by grafting affinity ligand, and many different types of agents (e.g., molecular drugs, genetic material) could be loaded together to enhance therapeutic efficacy. We used the MB-Lipo particles to deliver plasmid DNA to cancer cells as well as to primary cells, achieving transfection efficiency higher than that of a commercial agent. The particles also showed a high efficacy in cancer detection and treatment. In an animal study, we could target local tumor with MB-Lipo particles, which was confirmed by concurrent US imaging. Subsequent application of US flash released therapeutic materials 
in a site-specific and time-specific manner. Such treatments, benefitting from the efficient delivery and high potency (combination of anticancer drug and therapeutic gene) of MB-Lipo, enabled a significant reduction in tumor burden. All these results validated the dual use of MB-Lipo particles as a US contrast agent and a therapeutic delivery vehicle. Going forward, we envision that the developed MB-Lipo particles could have broader applications. The developed technology could promote early detection and treatment response monitoring, eventually informing personalized medicine strategies. The platform could also be used in other diseases (diabetes or arteriosclerosis), wherein US imaging is already widely employed. To faciliate the MB-Lipo's clinical translation, we plan to conduct the toxicology study as well as pre-clinical trial to evaluate its treatment efficacy.

\section{Supplementary Material}

Fig. S1 - Fig. S9.

http://www.thno.org/v04p1133s1.pdf

\section{Acknowledgements}

We thank Dr. Shao (MGH) for critically reviewing the manuscript. This work was funded through the National Research Foundation of Korea (the Basic Science Research Program 2010-0008846 to T.-J. Yoon and 2010-0009271 to H. J. Lee) and partially supported by a grant of O-song Medical Innovation Foundation R\&D project (HO13C0009) of Ministry of Health and Welfare in South Korea. H. Lee acknowledges a support by the National Institute of Health Grants, R01-HL113156. H. J. Jae also acknowledges a support by Seoul National University Hospital research fund (04-2012-0670).

\section{Conflict of Interests}

The authors have declared that no competing interest exists.

\section{References}

1. Kelkar SS, Reineke TM. Theranostics: combining imaging and therapy. Bioconjug Chem 2011; 22: 1879-1903.

2. Janib SM, Moses AS, Mackay JA. Imaging and drug delivery using theranostic nanoparticles. Adv Drug Deliv Rev 2010; 62: 1052-63.

3. Ansari C, Tikhomirov GA, Hong SH, Falconer RA, Loadman PM, Gill JH, Castaneda R, Hazard FK, Tong L, Lenkov OD, Felsher DW, Rao J, Daldrup-Link HE. Development of novel tumor-targeted theranostic nanoparticles activated by membrane-type matrix metalloproteinases for combined cancer magnetic resonance imaging and therapy. Small 2014; 10: 566-75.

4. Cho HS, Frangioni JV. Nanoparticles for biomedical imaging: fundamentals of clinical translation. Mol Imaging 2010; 9: 291-310.

5. Yoke R, Grulke E, MacPhail R. Metal-based nanoparticle interactions with the nervous system: the challenge of brain entry and the risk of retention in the organism. Wiley Interdiscip Rev Nanomed Nanobiotechnol 2013; 5: 346-73.

6. Yong KT, Law WC, Hu R, Ye L, Liu L, Swihart MT, Prasad PN. Nanotoxicity assessment of quantum dots: from cellular to primate studies. Chem Soc Rev 2013; 42: 1236-50.

7. Al-Jamal WT, Kostarelos K. Liposome: from a clinically established drug delivery system to a nanoparticle platform for theranostic nanomedicine. Acc Chem Res 2011; 44: 1094-104.
8. Nguyen AT, Wrenn SP. Acoustically active liposome-nanobubble complexes for enhanced ultrasonic imaging and ultrasound-triggered drug delivery. Wiley Interdiscip Rev Nanomed Nanobiotechnol 2014; 6: 316-25.

9. Hasik MJ, Kim DH, Howle LE, Needham D, Prush DP. Evaluation of synthetic phospholipid ultrasound contrast agents. Ultrasonics 2002; 40: 973-82.

10. Akbarzabeh A, Rezaei-Sadabady R, Davaran S, Joo SW, Zarghami N, Hanifehpour Y. Liposome: classification, preparation, and applications. Nanoscale Res Lett 2013; 8: 102-10.

11. Barenholz Y. Doxil® - the first FDA-approved nano-drug: lessons learned. J Control Release 2012; 160: 117-34.

12. Ferrara K, Pollard R, Borden M. Ultrasound mirobubble contrast agents: fundamentals and application to gene and drug delivery. Annu Rev Biomed Eng 2007; 9: 415-47.

13. Sirsi SR, Borden MA. Advances in ultrasound mediated gene therapy using microbubble contrast agents. Theranostics 2012; 2: 1208-22.

14. Klibanov AL, Shevchenko TI, Raju BI, Seip R, Chin CT. Ultrasound-triggered release of materials entrapped in microbubble-liposome constructs: A tool for targeted drug delivery. J Control Release 2010; 148: 13-7.

15. Yan F, Li L, Deng Z, Jin Q, Chen J, Yang W, Yeh CK, Wu J, Shandas R, Liu X, Zheng H. Paclitaxel-liposome-microbubble complexes as ultrasound-triggered therapeutic drug delivery carriers. J Control Release 2013; 166: 246-55.

16. Mitragotri S. Healing sound: the use of ultrasound in drug delivery and other therapeutic applicationis. Nat Rev Drug Discov 2005; 4: 255-60.

17. Geers B, Lentacker I, Sanders NN, Demeester I, Meairs S, De Smedt SC. Self-assembled liposome-loaded microbubbles: The missing link for safe and efficient ultrasound triggered drug-delivery. J Control Release 2011; 152: 249-56.

18. Kheirolomoom A, Dayton PA, Lum AF, Little E, Paoli EE, Zheng H, Ferrara KW. Acoustically-active microbubbles conjugated to liposomes: characterization of a proposed drug devliery vehicle. J Control Release 2007; 118: 275-84.

19. Dayton PA, Allen JS, Ferrara KW. The magnitude of radiation force on ultrasound contrast agents. J Acoust Soc Am 2002; 112: 2183-92.

20. Prentice P, Cuschieri A, Dholakia K, Prausnitz M, Campbell P. Membrane disruption by optically controlled microbubble cavitation. Nat Phys 2005; 1 : 107-10.

21. Manjappa AS, Chaudhari KR, Venkataraju MP, Dantuluri P, Nanda B, Sidda C, Sawant KK, Murthy RS. Antibody derivatization and conjugation strategies: application in preparation of stealth immunoliposome to target chemotherapeutics to tumor. J Control Release 2011; 150: 2-22.

22. Deng CX, Sieling F, Pan H, Cui J. Ultrasound-induced cell membrane porosity. Ultrasound Med Biol 2004; 30: 519-26.

23. Chang IS, Lee MW, Kim YI, Choi SH, Kim HC, Choi YW, Yoon CJ, Shin SW, Lim HK. Comparison between transauricular and transfemoral arterial for hepatic artery angiography in a rabbit model. J Vasc Interv Radiol 2011; 22: 1181-7.

24. Ninomiya $\mathrm{H}$. The vascular bed in the rabbit ear: microangiography and scanning electron microscopy of vascular corrosion casts. Anat Histol Embryol 2000; 29: 301-5.

25. Delalande A, Kotopoulis S, Postema M, Midoux P, Pichon C. Sonoporation: Mechanistic insights and ongoing challenges for gene transfer. Gene 2013; 525: 191-9.

26. Noble ML, Kuhr CS, Graves SS, Loeb KR, Sun SS, Keilman GW. Ultrasound-targeted microbubble destruction-mediated gene delivery into canine livers. Mol Ther 2013; 21: 1687-94.

27. Zhou Y, Kumon RE, Cui J, Deng CX. The size of sonoporation pores on the cell membrane. Ultrasound Med Biol 2009; 35: 1756-60.

28. Arangoa MA, Düzgünes N, Tros de llarduya C. Increased receptor-mediated gene delivery to the liver by protamine-enhanced-asialofetuin-lipoplexes. Gene Ther 2003; 10: 5-14

29. Inho Y, Furuno T, Hirashima N, Kitamoto D, Nakanishi M. Synergistic effect of a biosurfactant and protamine on gene transfection efficiency. Eur J Pharm Sci 2013; 49: 1-9.

30. Gabizon A, Shmeeda H, Grenader T. Pharmacological basis of pegylated liposomal doxorubicin: impact on cancer therapy. Eur J Pharm Sci 2012; 45: 388-98.

31. Haran G, Cohen R, Bar LK, Barenholz Y. Transmembrane ammonium sulfate gradients in liposomes produce efficient and stable entrapment of amphipathic weak bases. Biochim Biophys Acta 1993; 1151: 201-15.

32. Lee C, Dhillon J, Wang MY, Gao Y, Hu K, Park E, Astanehe A, Hung MC, Eirew P, Eaves CJ, Dunn SE. Targeting YB-1 in HER-2 overexpressing breast cancer cells induces apoptosis via the mTOR/STAT3 pathway and suppresses tumor growth in mice. Cancer Res 2008; 68: 8661-6.

33. Kang Y, Park MA, Heo SW, Park SY, Kang KW, Park PH, Kim JA. The radio-sensitizing effect of xanthohumol is mediated by STAT3 and EGFR suppression in doxorubicin-resistant MCF-7 human breast cancer cells. Biochim Biophys Acta 2013; 1830: 2638-48.

34. Choi YJ, Li X, Hydbring P, Sanda T, Stefano J, Christie AL, Signoretti S, Look AT, Kung AL, von Boehmer $\mathrm{H}$, Sicinski P. The requirement for cyclin D function in tumor maintenance. Cancer Cell 2012; 22: 438-51.

35. Dubik D, Dembinski TC, Shiu RP. Stimulation of c-myc oncogene expression associated with estrogen-induced proliferation of human breast cancer cells. Cancer Res 1987; 47: 6517-21.

36. Stennicke HR, Salvesen GS. Biochemical characteristics of caspases-3, -6, -7, and -8. J Biol Chem 1997; 272: 25719-23. 
37. Sabine VS, Faratian D, Kirkegaard-Clausen T, Bartlett JM. Validation of activated caspase-3 antibody staining as a marker of apoptosis in breast cancer. Histopathology 2012; 60: 369-71.

38. Luo W, Zhou X, Zheng X, He G, Yu M, Li Q. Role of sonography for implantation and sequential evaluation of a VX2 rabbit liver tumor model. J Ultrasound Med 2010; 29: 51-60.

39. Prabhakar U, Maeda H, Jain RK, et al. Challenges and key considerations of the enhanced permeability and retention effect for nanomedicine drug delivery in oncology. Cancer Res 2013; 73: 2412-7.

40. Chow EK, Ho D, Cancer nanomedicine: from drug delivery to imaging. Sci Transl Med 2013; 5: 216rv4.

41. Bertrand N, Wu J, Xu X, Kamaly N, Farokhzad OC, Cancer nanotechnology: the impact of passive and active targeting in the era of modern cancer biology. Adv Drug Deliv Rev 2014; 66: 2-25. 